\title{
No future forever. L'interartialité et l'intermédialité post-punk dans le roman français contemporain
}

\author{
Eva Voldřichová Beránková
}

Faculté des Lettres, Université Charles (Prague)*

L'objectif du présent article consiste dans une réflexion sur le rôle joué par la musique et le cinéma post-punk dans l'œuvre poétique de Virginie Despentes et Michel Houellebecq, ainsi que sur l'ébauche de nouvelles approches herméneutiques qu'une telle littérature semble requérir. Le texte met en rapport les expérimentations littéraires et musicales de la fin du dix-neuvième siècle avec le foisonnement post-punk contemporain, décelant dans les deux mouvances un potentiel de subversion, de créativité et d'exploration inespéré. Il résume les carrières interartiales des deux artistes étudiés, met l'accent sur les similitudes entre leurs pratiques d'une « littérature du concret » et évalue leur portée internationale, ainsi que leur influence sur de nouvelles générations de lecteurs et spectateurs.

Mots-clés : interartialité, intermédialité, roman français contemporain, (post) punk, Virginie Despentes, Michel Houellebecq.

\section{Le foisonnement créatif des fins de siècles}

Les charnières de siècles se ressemblent. Les symbolistes et décadents français avaient jadis adopté le verlainien « de la musique avant toute chose » (Verlaine 1884 : 23), poussant la provocation du bourgeois jusqu'à jeter les bases d'une véritable « littérature wagnérienne » (Bertrand et al. 1996 : 16), dotée d'une temporalité éclatée, révolutionnaire, musicale, complètement affranchie de l'histoire événementielle ainsi que de la réalité objective et tournée davantage vers l'exploration des états intérieurs d'un moi déchiré. Pierre Boulez note qu'à l'époque, dans l'œuvre debussiste, tout comme dans le roman symboliste contemporain, l'être se

\footnotetext{
Eva.Berankova@ff.cuni.cz

Le présent article s'inscrit dans le Projet Européen du Développement Régional « Créativité et adaptabilité comme conditions du succès de l'Europe dans un monde interconnecté »
}

(No. CZ.02.1.01/0.0/0.0/16_019/0000734). 
trouve pour la première fois "spontanément relié à lui-même 》 (Caullier 1983 : 148), dans l'instant présent où il se trouve. Valérie Michelet Jacquod désignera ce phénomène comme un « art de l'extrême conscience » (Michelet Jacquod 2008).

De son côté, Stravinski parviendra à un but semblable en restaurant une communauté d'expression et en recréant une musique tellurique, primitive, rituelle, où l'individu n'existe plus (ou pas encore), mais qui est susceptible de mettre en valeur «l'homme, unique, dans toute son éternité » (Boucourechliev $1982: 128)$.

Tandis que la critique officielle avait pris l'habitude de se déchaîner contre les dangers esthétiques, politiques ou moraux de la folle synesthésie décadente, les écrivains fin-de-siècle reconnaissaient dans la musique (que cela ait été le romantisme de Wagner' ${ }^{1}$ l'impressionnisme de Debussy ou les expérimentations modernistes de Stravinsky) un potentiel de subversion, de créativité et d'exploration inespéré. Loin de déboucher sur une fin des temps imminente, la décadence n'a ainsi été qu'une étape préliminaire à la grande mutation du monde moderne. En effet, obsédée par l'idée de son propre déclin, la société de l'époque n'a souvent perçu que le côté morbide voire grotesque de la production littéraire et musicale fin-de-siècle, sans saisir leur incroyable force de rénovation et la vitalité des nouvelles conceptions du monde que les écrivains et musiciens du dix-neuvième siècle finissant proposaient au public.

Cent ans plus tard, leurs successeurs contemporains ne sont pas en reste, cherchant leur propre voie vers une poétique teintée de lyrisme noir. Et, une fois de plus, ils doivent faire face à une certaine méfiance des milieux académiques effrayés. Des romanciers tels que Virginie Despentes (1969), Michel Houellebecq (1956) et, dans une moindre mesure, Maurice G. Dantec (1959-2016) ou Vincent Ravalec (1962), naviguent avec hardiesse entre le roman philosophique, la science-fiction et le cyberpunk. Depuis les années 1990, ils remettent le scandale à l'ordre du jour, abordant sans complexes des sujets sulfureux, voire obscènes, liés à la vie de la génération $\mathrm{X}$ (prostitution volontaire, pornographie, viol, toxicomanie, terrorisme, etc.). Fréquemment accusés de provocation facile, marginalisés ou censurés au début, ils se sont pour la plupart « assagis » depuis, devenant lauréats du prix Goncourt (Michel Houellebecq avec La Carte et le Territoire en 2010) voire membres de l'académie décelant ce même prix (Virginie Despentes en 2016). L'objet de la présente contribution sera une courte réflexion sur le rôle joué par la musique et le cinéma (post-)punk dans l'œuvre poétique de Virginie Despentes et Michel Houellebecq et sur l'ébauche de nouvelles approches herméneutiques qu'une telle littérature semble requérir.

\footnotetext{
${ }^{1}$ Sur la puissance suggestive de la musique selon Wagner, dont la conception artistique - l'alliance intime de la poésie et de la musique - a influé sur celle de Baudelaire, voir Katalin Bartha-Kovács (2015).
} 


\section{L’histoire du (post-)punk}

Commençons par une brève récapitulation historique : le post-punk est d'abord un genre musical né vers la fin des années $1970^{2}$. Il prolonge la vague du punk radical, tout en se différenciant de son prédécesseur par davantage d'introversion, par une nette propension à l'humour sarcastique, aux paradoxes et au second degré (Marcus 2000), ainsi que par un goût prononcé pour l'expérimentation et une plus grande élaboration, voire sophistication dans la production musicale. Le son devient plus « arty », par rapport aux performances directes et violentes du premier punk.

La philosophie générale des artistes rattachés à la mouvance post-punk se trouve résumée par cette déclaration d'Allen Ravenstine de Pere Ubu [remarquons au passage le petit clin d'œil à Alfred Jarry] en 1978 : «Les Sex Pistols chantent No Future, mais il y a un futur et nous essayons de le construire. " (Reynolds 2007 : 15) Un autre reproche, à la fois existentiel et politique, que les musiciens de l'époque formulent à l'égard du mouvement punk originel est le fait que ce dernier s'est vu vite récupéré par l'industrie musicale et n'est ainsi devenu qu'une « parodie inoffensive de lui-même » (Ibidem).

Le post-punk a joué un rôle important dans la scène musicale indépendante des années 1980, contribuant à la gestation de plusieurs courants majeurs du rock, dont le rock gothique, le rock industriel, le rock indépendant ou le rock alternatif. Or, le terme a fini par désigner en même temps des groupes aux productions beaucoup plus commerciales et légères, comme l'« italo disco » (orienté dancefloor et destiné aux discothèques), les Nouveaux-Romantiques, le synthpop, la new wave, la cold wave, la noise pop, le shoegazing, la scène baggy à Manchester, celle du trip-hop à Bristol ou les innombrables branches de l'electro. Décidément, il y a aujourd'hui autant de mouvances post-punk qu'il n'y avait de cafés-concerts symbolico-décadents dans le Paris de la fin du XIX siècle et la "pérennité » de ces groupes ainsi que des manifestes lancés par eux (valables de quelques mois à quelques années) s'avère, elle aussi, tout à fait comparable.

Avant de tirer quelques conclusions herméneutiques et didactiques d'un tel foisonnement à la fois créatif et substantiellement instable, revenons à nos deux artistes et à leurs activités intermédiales. En effet, en dehors de leurs carrières d'écrivains, tant Despentes que Houellebecq sont aussi, à l'instar de tous les postpunks, des musiciens, cinéastes et performeurs redoutables.

\footnotetext{
${ }^{2}$ L'historien Clinton Heylin situe ses débuts entre août 1977 et mai 1978 avec l'arrivée du guitariste John McKay au sein de Siouxsie and the Banshees, la formation des groupes Magazine et Public Image Ltd, et la décision de Wire de passer d'un quintet à un quartet en éliminant toutes les parties superflues de leurs morceaux. (Heylin 2006 : 460)
} 


\section{Les parcours de Virginie Despentes et de Michel Houellebecq}

Punk dès l'âge de treize ans, Virginie Despentes a travaillé pendant plusieurs années pour un label de disques appelés Gougnaf. Dans les années 1990, elle a rappé dans le groupe Straight Royer, s'est exhibée dans un peep-show et a traversé une longue et éprouvante période « prostitution, drogues et post-punk » qui a fini par l'orienter vers la littérature (Médioni 1996). Même après sa consécration officielle par les maisons d'édition, elle n'a pas renoncé à la musique. En 2003, elle a adapté en français la chanson « Protect Me from What I Want » du groupe de rock alternatif britannique Placebo. Deux années plus tard, elle a écrit trois chansons pour le groupe de rock français A. S. Dragon : Cher tueur, Seule à Paris et Cloue-moi au ciel. En 2006, elle a servi de disc-jockey lors du happening « Explicit part 1 Yesporno », une manifestation organisée autour de la féminité et de la pornographie. Il arrive encore assez souvent à Virginie Despentes de chanter elle-même, accompagnée de son groupe de trip-hop baptisé Skywalker pour lequel elle écrit des paroles de chansons inspirées par les œuvres de Stephen King, Hubert Selby ou Abel Ferrara.

Pour ce qui est du cinéma, Despentes a réalisé quatre films indépendants : Baise-moi (2000), un long métrage à scandale, tourné en collaboration avec Coralie Trinh Thi ; Mauvaise étoile (2006), un clip musical psychédélique de quatre minutes et demie pour Patrick Eudeline ; Mutantes (Féminisme Porno Punk) (2009), une série d'entretiens punk avec vingt-quatre intellectuels, écrivains, théoriciens et militants queer « sur le féminisme pro-sexe et la post-pornographie », et finalement Bye Bye Blondie (2011), un long métrage de fiction inspiré de son roman éponyme. De plus, elle a contribué aux adaptations de deux autres de ses romans : Les Jolies Choses (2001, réalisé par Gilles Paquet-Brenner) et Teen spirit (2007, réalisé par Olivier de Plas sous le titre Tel père telle fille). Avec Béatrix Dalle, elle a récemment joué dans Une Charogne (2018) de Renaud de Foville, après être brièvement apparue dans L'Érotisme vu par Christine Angot (2001, réalisé par Laetitia Masson), Les Travailleu(r)ses du Sexe (2010, documentaire de Jean-Michel Carré) et Becs et Ongles (2010, court métrage d'Élodie Monlibert).

Michel Houellebecq, un ancien du punk lui aussi ${ }^{3}$, a sorti trois disques d'une sorte de « rap mou », comme Bertrand Burgalat a défini le style de ses performances (Wolfe 1999) : Le Sens du combat (1996), où il lit ses poèmes du recueil éponyme sur fond de musique improvisée ; Présence humaine (2000), où il chante certains de ses textes sur une musique de Bertrand Burgalat, et Éta-

\footnotetext{
3 « [Le punk], oui, j'aimais bien, c'est même le dernier mouvement que j'ai suivi. » (Beauvallet $2000: 38-41)$
} 
blissement d'un ciel d'alternance (2007), une nouvelle lecture de poèmes avec accompagnement musical du compositeur Jean-Jacques Birgét.

Il a réalisé quatre films : Cristal de souffrance (1978), un court métrage muet, en noir et blanc ; Déséquilibres (1982), une fiction sur une femme handicapée qui reconnaît l'homme qui l'avait jadis poussée d'un pont et l'avait ainsi clouée sur un fauteuil roulant ; La Rivière (2001), une curieuse ode au lesbianisme dans un monde post-apocalyptique d'où les mâles ont disparu, et La Possibilité d'une île (2008), un long métrage d'anticipation tiré de son roman éponyme. Michel Houellebecq a également écrit les scénarii de deux autres films : l'Extension du domaine de la lutte (1999) de Philippe Harel et le Monde extérieur (2004) de David Rault.

Il est apparu dans un certain nombre de documentaires ${ }^{5}$ et a interprété plusieurs rôles dans des films de fiction. Guillaume Nicloux l'a ainsi engagé dans le rôle de Bernard Gérard dans L'Affaire Gordji : Histoire d'une cohabitation (2012) et, deux années plus tard, l'écrivain a joué lui-même dans L'Enlèvement de Michel Houellebecq (2014) du même réalisateur. En 2014, il a interprété le rôle de Paul dans Near Death Experience de Gustave Kervern et Benoît Delépine et en 2016 celui du propriétaire de la maison d'hôte dans Sainte-Amour du même Benoît Delépine.

\section{Pour une poétique nouvelle}

La première remarque qui s'impose donc à tous ceux qui voudraient analyser l'œuvre despentienne ou houellebecquienne consiste à rappeler l'interartialité et l'intermédialité évidentes de leurs subcultures spécifiques, de cette fameuse " poésie punk » sous-jacente qui ignore superbement les classements génériques ainsi que toute hiérarchie d'arts ou de média.

Au cours d'un entretien qui a suivi la sortie de son second disque, Michel Houellebecq déclare sans ambages :

Un disque, ce n'est pas dérisoire du tout. J'ai toujours lu et écouté des disques : à 15 ans, je me passionnais autant pour Jimi Hendrix que pour Dostoïevski. Il n'y a pas de différence, toujours pas de hiérarchie. Un livre n'est pas une chose plus sérieuse, un disque ne se démode pas. C'est plus délicat pour le cinéma : là, oui, je trouve que la littérature, c'est quand même plus sérieux. 2001 : l'odyssée de l'espace, je trouve que ça a mal vieilli. Je n'ai jamais ressenti une telle déception par rapport à un disque. (Beauvallet $2000: 38$ )

\footnotetext{
${ }^{4}$ De nombreux poèmes de Michel Houellebecq ont été mis en musique par d'autres interprètes : Yvan Hio, le groupe Helium Vola, Carla Bruni ou Jean-Louis Aubert.

${ }^{5}$ À titre d'exemple, nous pouvons mentionner L'Érotisme vu par Christine Angot (2001) de Laetitia Masson ; Plateforme. Une lecture (2002) de Michel Meyer ; Houellebecq, entretien (2005) de Christian Fevret ou To Stay Alive: A Method (2016) d'Erik Lieshout, Arno Hagers et Reiner von Brummelen.
} 
Virginie Despentes, elle aussi, met l'accent sur le foisonnement interartial qui a marqué sa jeunesse :

J'ai grandi chez les punks, coupée du reste du monde, à l'abri, avec ce code hyper strict punk, en prenant cette culture très au sérieux, en misant tout là-bas [...] On avait vu les mêmes films, lu les mêmes bouquins, écouté les mêmes groupes au même moment. Des films comme Orange mécanique, Scarface, Mad Max ou L'Exorciste. [...] Côté bouquins, c'était Ellroy ou le livre de Mesrine, tout le truc gauche dure. (Bourmeau $1996: 28$ )

Inutile donc de vouloir pratiquer toute « critique immanente » et d'opérer des coupures méthodologiques structuralistes entre la littérature et la musique, la musique et le cinéma, le cinéma et la performance dans un milieu où une chanson inspire un roman qui est par la suite adapté à l'écran et dont des extraits peuvent être tant mis en musique que martelés lors d'une manifestation politique. Comme Jérôme Meizoz le rappelle,

la posture "Houellebecq" consiste à rejouer machinalement dans l'espace public le personnage d'antihéros aux propos "socialement inacceptables" auquel il a délégué la narration. Par un étrange renversement, la conduite de fiction (les propos du narrateur) précède souvent la conduite sociale (ceux de la posture auctoriale) et semble la générer. (Meizoz 2004 : 202)

Autrement dit, si les cinq minutes de silence admiratif qui suivent un concert de Mozart sont encore du Mozart, le scandale, voire le procès judiciaire, qui suit la publication d'un roman de Michel Houellebecq, c'est encore du Houellebecq.

Ce mélange d'arts, de médias et de genres s'accompagne d'une profusion de langues et de cultures. Les textes de Houellebecq, comme ceux de Despentes, sont truffés de références internationales, allant de la philosophie classique à la culture pop. Les œuvres des deux écrivains s'exportent, elles aussi, très bien, bénéficiant d'un accueil enthousiaste de la part de la critique anglo-saxonne, allemande ou slave. Michel Houellebecq a été traduit en quarante-cinq langues (Estier 2017), de sorte qu'il est aujourd'hui considéré comme le plus « globalisé » des écrivains français contemporains. D'ailleurs, ne s'est-il pas réfugié en Thaïlande et à Cuba pour écrire Soumission, après avoir travaillé sur d'autres livres en Espagne, en Irlande ou sur Lanzarote ? Virginie Despentes, elle, traduit couramment de l'anglais et plus de trois quarts d'études académiques la concernant sont aujourd'hui, elles aussi, rédigées dans cette langue, sous le label « gender studies » pour la plupart. The Guardian a pris l'habitude de surnommer l'écrivaine la « rock'n'roll Zola » de notre temps (Chrisafis 2018). 
Un autre point commun des deux romanciers consiste dans leur attachement à une littérature du concret, presque balzacienne, qui s'efforce de « dire le monde $^{6} »$. Michel Houellebecq ne cesse d'affirmer son refus de l'art pour l'art, du style pour le style :

Je n'ai jamais pu, pour ma part, assister sans un serrement de cœur à la débauche de techniques mises en œuvre par tel ou tel « formaliste-Minuit» pour un résultat aussi mince. Pour tenir le coup, je me suis souvent répété cette phrase de Schopenhauer : « La première - et pratiquement la seule - condition d'un bon style, c'est d'avoir quelque chose à dire. » (Houellebecq $1997:$ 178)

L'éditrice Marion Mazauric résume cette nouvelle tendance littéraire ainsi :

À la fin des années 1980, un vrai basculement s'opère avec l'émergence d'une nouvelle génération d'écrivains qui retrouvent le roman comme moyen de dire le réel. Après Echenoz, Ernaux, Belletto, etc., Djian est le premier à rencontrer un immense succès. Mais il lui aura fallu plus de dix ans pour enfin acquérir une reconnaissance critique. Puis arrivent Holder, Ravalec, Houellebecq, Beigbeder, Gunzig, etc. Une des très grands est sans doute Virginie Despentes, qui « littérarise » la langue orale et la violence sociale comme personne. On oublie souvent que ces auteurs sont issus des classes moyennes et des contre-cultures. Ils portent un regard décalé sur notre présent, un regard d'historiens, souvent ironique, souvent aussi noir que le spectacle du monde. (Rousset 2005)

Le sociologue Éric Fassin va encore plus loin, lorsqu'il classe Houellebecq et Despentes parmi les auteurs d'une « littérature performative » dont la tâche consisterait à « construire le monde » (Ibidem.). Les deux auteurs semblent participer d'un mouvement plus global de dénonciation et de rejet du libéralisme dont les contours sont mouvants : altermondialistes, gays-lesbiennes, sans-papier, anti-OGM, postpunks, web-militants... Loin de se résumer à des jeux formels, leurs textes sont censés mobiliser, pointer les différentes crises et problématiques occidentales, que ce soit le clonage humain, les désordres climatiques, la pollution nucléaire, la migration massive, la crise du logement, la violence entre les sexes, etc. Selon Houellebecq et Despentes, un écrivain doit « prendre en charge le monde dans ses œuvres » (Weitzmann 1996 : 56), ce qui représente d'ailleurs une ambition similaire à celle des grands rockers ou punkers du passé (Bourmeau 1996 : 52).

\footnotetext{
${ }^{6}$ Littérature que Michel Houellebecq met toujours en rapport avec la musique : « Ce n'est pas facile, en littérature, de faire un personnage intéressant avec quelqu'un qui ne l'est pas du tout, de manier l'ordinaire. Moi, je trouve que j'y arrive bien, c'est même la seule chose exceptionnelle dans mes livres. Sur des textes courts, dans le rock, des moments de vie ordinaire peuvent très bien passer. Par exemple, j'ai toujours beaucoup aimé les paroles de She's leaving home des Beatles : l'histoire d'une fille qui quitte une famille ordinaire anglaise. Le rock a toujours fait ça très bien, ces petites chroniques. La littérature a changé, mais pendant des années, elle a eu du mal à raconter les vies de base, dans leur aspect social, avec les petits faits, les incidents. » (Beauvallet $2000: 39$ )
} 


\section{Conclusion}

En effet, au croisement des avant-gardes artistiques, de la contre-culture et de la pensée libertaire, le (post-)punk s'inscrivait dès le début dans la perspective d'un mouvement d'émancipation. Comme Fabien Hein le rappelle : «Être punk se vit. Concrètement. Sans différé. Sans l'aval de quiconque. Par l'engagement et par l'action. Avec l'autonomie et l'indépendance pour l'horizon. L'émancipation pour élan. L'autodétermination pour tout bagage. » (Hein 2012 : 132)

En effet, au-delà de la provocation facile et du trash, le punk est profondément lié à la culture DIY (Do It Yourself!), au principe d'agir sans le soutien institutionnel, sans l'État et sans le capital. Tout un chacun est incité par lui à monter sur scène pendant un concert, créer son propre groupe, écrire dans un fanzine. Rompant avec une culture qui repose sur le spectacle, la passivité et la consommation, il milite pour une démocratisation des pratiques culturelles. Comme jadis dans les cabarets fin-de-siècle ou, plus tard, au sein du mouvement Dada, tout le monde peut devenir créateur. Encourageant l'autoproduction ainsi que la culture participative, libérant la créativité et les désirs, selon le journaliste Greil Marcus, le punk procède d'un « besoin urgent de vivre non pas comme objet mais comme sujet de l'histoire - de vivre comme si quelque chose dépendait réellement de notre propre action » (Marcus $2000: 14$ ).

Dans la trilogie de Vernon Subutex, Virginie Despentes esquisse la possibilité d'une telle société alternative, fondée sur la communion musicale (les fameuses « convergences »), tandis que Michel Houellebecq fait échapper ses héros à la morne vie des célibataires parisiens en brodant des fantasmes autour du clonage, du tourisme sexuel, de l'islamisation ou de l'immortalité scientifique. Ceci toujours dans le même esprit de bricolage (ou « Do It Yourself ») qui caractérise les héros despentiens.

Pour conclure, je voudrais exprimer deux souhaits : tout d'abord que Michel Houellebecq et Virginie Despentes soient un jour enseignés au même titre que les « poètes maudits » du XIX ${ }^{\mathrm{e}}$ siècle finissant, avec lesquels ils partagent le même goût de la " beauté convulsive " et le même amour de la provocation. Ensuite, que les méthodes herméneutiques et didactiques de l'avenir soient du moins un peu « punk », à savoir susceptibles de stimuler la spontanéité, la créativité et l'auto-expression des étudiants.

La critique littéraire Marianne Payot a jadis conclu un article consacré à Virginie Despentes par cette remarque perspicace : «La scandaleuse s'impose comme la chef de file d'une génération gaiement libertaire et décomplexée. » (Payot 2010) Qu'est-ce qu'un post-punk (ou un prof de littérature) d'aujourd'hui pourrait espérer de plus? 


\section{Références bibliographiques}

Bartha-Kovács 2015 : Katalin Bartha-Kovács, « Le sentiment, l’invisible de la peinture à l'épreuve du visible : le colorisme de Delacroix à travers la critique d'art de Baudelaire », Ostium. Revue des sciences humaines, ${ }^{\circ} 2,109-120$.

Beauvallet 2000 : J. D. Beauvallet, «Extension du domaine de la flûte », entretien avec Michel Houellebecq, Les Inrockuptibles, $\mathrm{n}^{\circ}$ 240, 25 avril, 38-41.

Bertrand et al. 1996 : J. P. Bertrand, M. Biron, J. Dubois, Jeannine Pâque, Le roman célibataire. D'À rebours à Paludes, Paris : José Corti.

Boucourechliev 1982 : A. Boucourechliev, Stravinsky, Paris : Fayard.

Bourmeau 1996 : S. Bourneau, « Lis-moi », Les Inrockuptibles, n $^{\circ}$ 50, 27 mars, 28-30.

Caullier 1983 : J. Caullier, « Musique et Décadence», Romantisme, n 42, « Décadence», 137-150.

Champenois 1999 : S. Champenois, « Mutant mutandis », Libération, 9 avril, 52.

Chrisafis 2018 : A. Chrisafis, "What is going on in men's heads when women's pleasure has become a problem?", interview with Virginie Despentes. <https://www. theguardian.com/books/2018/aug/31/virginie-despentes-interview-baise-moi-vernon-subutex>. 12/01/2019.

Estier 2017 : S. Estier, « Houellebecq polyglotte », Fabula / Les colloques, Les « voix » de Michel Houellebecq, 8 mars. $<$ http://www.fabula.org/colloques/document 4351. php>. 12/01/2019.

Hein 2012 : F. Hein, Do it yourself! Autodétermination et culture punk, Lyon : Le passager clandestin.

Heylin 2006 : C. Heylin, Babylon's Burning: From Punk to Grunge, Penguin Books Ltd.

Lacoche 2001 : P. Lacoche, « Des romans français taillés dans le rock », Magazine littéraire, $\mathrm{n}^{\circ} 404$, dossier « Les écrivains rock », décembre, 26-29.

Marcus 2000 : G. Marcus, Lipstick traces. Une histoire secrète du vingtième siècle, traduit de l'anglais par Guillaume Godard, Paris : Éditions Allia.

Médioni 1996 : G. Médioni, « Despentes abrupte !», L’Express, 18 avril. <https://www. lexpress.fr/informations/despentes-abrupte_613620.html>. 12/01/2019.

Meizoz 2004 : J. Meizoz, L'œil sociologue et la littérature, Genève : Slatkine.

Michelet Jacquod 2008 : V. Michelet Jacquod, Le Roman symboliste : un art de l'extrême conscience : Edouard Dujardin, André Gide, Remy de Gourmont, Marcel Schwob, Genève : Droz.

Noguez 2003 : D. Noguez, Houellebecq, en fait, Paris : Fayard.

Payot 2010 : M. Payot, « Virginie Despentes décape à vif », L'Express, 19 août. <https:// www.lexpress.fr/culture/livre/apocalypse-bebe_913288.html>. 12/01/2019.

Reynolds 2007 : S. Reynolds, Rip It Up and Start Again. Post-punk (1978-1984), traduit de l'anglais par Aude de Hesdin et Étienne Menu, Paris : Allia.

Rousset 2005 : M. Rousset, « Houellebecq, les possibilités d'un livre », table ronde avec Éric Fassin, Marion Mazauric et Isabelle Lorand. <http://www.regards.fr/accespayant/archives-web/houellebecq-les-possibilites-d-un,2138>. 12/01/2019.

Verlaine 1884 : P. Verlaine, «Art poétique », Jadis et Naguère : poésies, Paris : Léon Vanier. 
Weitzman 1996 : M. Weitzman, « J'ai plus que des doutes », Les Inrockuptibles, n 52, 19 avril, $56-59$.

Wolfe 1999 : M. Wolfe, « Michel Houellebecq », République des Lettres, 15 août. <https:// republique-des-lettres.fr/689-michel-houellebecq.php>. 12/01/2019.

\section{Eva Voldržihova Berankova}

\section{No future forever. Post-pank, interumetničko i intermedijalno u savremenom francuskom romanu}

Cilj ovog rada jeste da predstavi razmišljanja o ulozi post-pank muzike i filma u poetskom stvaralaštvu Viržinije Depant i Mišela Uelbeka, kao i o nacrtu za nove hermeneutičke postupke koje takva književnost, čini se, zahteva. Tekst povezuje književna i muzička istraživanja s kraja XIX veka sa bogatstvom savremenog post-panka, otkrivajući da obe ove tendencije sadrže neočekivani potencijal za subverzivnost, kreativnost $\mathrm{i}$ istraživanje. U tekstu je predstavljen pregled interumetničke karijere ova dva autora, ističu se sličnosti njihovih postupaka karakterističnih za „književnost konkretnog“, kao i njihov uticaj na nove generacije čitalaca i gledalaca.

Ključne reči: interumetničko, intermedijalno, savremeni francuski roman, (post) pank, Viržinija Depant, Mišel Uelbek. 